\title{
ASPECTOS CONCEITUAIS E METODOLÓGICOS DE REDES SOCIAIS E SUA INFLUÊNCIA NO ESTUDO DE FLUXOS DE INFORMAÇÃO
}

\author{
Cibele Roberta Sugahara \\ Waldomiro Vergueiro
}

\section{Resumo:}

As ligações e interações propiciadas pelas redes sociais permitem compreender como ocorrem os fluxos de informação entre indivíduos e instituições que unem esforços na busca de metas comuns. $\mathrm{O}$ artigo apresenta aspectos conceituais sobre redes e redes sociais ressaltando que a estrutura e as relações de interação e intermediação entre os elos da rede impulsionam mudanças nos fluxos de informação. Descreve a metodologia de Análise de Redes Sociais (ARS) sinalizando como esta pode ser utilizada na área da Ciência da Informação para compreender os fluxos de informação que se configuram e re-configuram nas redes sociais a partir da estrutura de relacionamento.

\section{Palavras-chave:}

Fluxos de informação; Redes sociais; Análise de redes sociais

\section{CONCEPTUAL AND METHODOLOGICAL ASPECTS OF SOCIAL NETWORKS AND ITS INFLUENCE ON INFORMATION FLOW STUDIES}

\begin{abstract}
:
The links and interactions offered by the social networks allow the understanding of how information flows occur among individuals and institutions that join forces to pursuit common goals. The paper presents conceptual aspects of networks and social networks, emphasizing that the structure and the interaction and intermediation relationship among the links of the network drive changes in information flow. Describes the Analysis of Social Networks (ARS) methodology, indicating how it can be used in the Information Science to understand the information flows, that shape and re-shape the social networks, starting from the structure of relationships.
\end{abstract}

\section{Keywords:}

Information flows; Social networks; Social networks analysis 


\section{INTRODUÇÃO}

Em período recente, a troca de informações em redes de grupos sociais delimitados pelo uso da metodologia da análise de redes sociais tem sido discutida na área da Ciência da Informação. Neste artigo, pode-se entender o conceito de rede como compreendendo um conjunto de atores que apresentam ligação direta ou indireta em seus relacionamentos, que, nas Ciências Sociais, são representados por sujeitos sociais - indivíduos, grupos, organizações - conectados por algum tipo de relação, valor ou interesse compartilhado (ALBAGLI; MACIEL, 2004; MARTELETO, 2001; TOMAÉL, 2008; TOMAÉL et al., 2005). Dessa forma, as características estruturais da rede e a posição estratégica de seus atores em relação aos outros atores permitem identificar e compreender quais deles atuam como fonte de informação para a execução das atividades de cada um e como tal estrutura afeta os fluxos de informação.

Nas Ciências Sociais, as redes podem evidenciar os movimentos institucionalizados, ao reunir indivíduos e grupos em uma associação, sendo formada por indivíduos, grupos ou organizações. Sua dinâmica está voltada para a perpetuação, consolidação e o desenvolvimento das atividades dos seus integrantes (MARTELETO, 2001). Por sua vez, o tema fluxo de informação tem estreita identidade com a área da Ciência da Informação, uma vez que esta objetiva investigar as propriedades e o comportamento da informação, as forças que governam o seu fluxo e os meios de processá-la para ótima acessibilidade e uso (SHERA; CLEVELAND, 1977).

Este artigo apresenta conceitos e observações sobre fluxos de informação em redes tendo em vista seu caráter social que acaba alimentando o desenvolvimento do conhecimento coletivo. Parte-se do pressuposto de que as interações sociais propiciam a formação de redes com pontos de convergência para a troca de informação. Desta forma, serão abordadas algumas questões fundamentais sobre a metodologia de Análise de Redes Sociais que podem corroborar para a compreensão das relações de troca de informação e colaboração entre os elos e sua representação visual. Entende-se que a descrição de métricas e medidas desta metodologia pode sinalizar como identificar o papel que cada elo desempenha de acordo com sua posição estrutural e sua influência no fluxo de informação junto às estruturas sociais.

(c) Revista Digital de Biblioteconomia e Ciência da Informação,Campinas, v.7, n. 2, p. 102-117, jan./jun. 2010- ISSN: 1678-765X. 


\section{REDES SOCIAIS: MARCO REFERENCIAL}

A organização social em redes já existia em outros tempos e espaços, mas como enfatiza Castells (1999) com a evolução social associada à tecnologia da informação uma nova base material está sendo construída em rede definindo os processos sociais predominantes e dando forma à própria estrutura social.

Para iniciar as reflexões sobre redes são oportunas as observações de Castells (1999, p. 498) ao definir rede como "um conjunto de nós interconectados”, sendo o nó o ponto no qual uma curva se entrecorta. O que é um nó depende do tipo de redes concretas de que falamos. Por consequência, os nós possibilitam observar os laços ou conectividades existentes entre os integrantes das redes.

Na estrutura social em rede as conexões podem funcionar como fontes de orientação e desorientação das sociedades. Como uma forma global de organização, a rede tem por princípio a participação individual e dos seus pares como base para seu delineamento e expansão.

Castells (1999, p. 497) afirma que a lógica de redes traz uma determinação social em nível mais alto que a dos interesses sociais específicos, expressos por meio delas: “o poder dos fluxos é mais importante que os fluxos de poder”. Neste sentido, as conexões que ligam as redes, como, por exemplo, os fluxos de informação representam instrumentos privilegiados de poder.

Ainda de acordo com Castells (1999, p. 498) as redes são “estruturas abertas capazes de expandir de forma ilimitada, integrando novos nós desde que consigam comunicar-se dentro da rede, ou seja, desde que compartilhem os mesmos códigos de comunicação”. Em concordância com esse posicionamento, Marteleto (2001, p. 72) considera que a rede social representa “[...] um conjunto de participantes autônomos, unindo idéias e recursos em torno de valores e interesses compartilhados”. Assim, conclui-se que as relações de interação entre os indivíduos de uma rede social oportunizam a troca e o compartilhamento de informação e conhecimento.

(c) Revista Digital de Biblioteconomia e Ciência da Informação,Campinas, v.7, n. 2, p. 102-117, jan.jjun. 2010- ISSN: 1678-765X. 
Mais do que isso: como assinala Tomaél (2008, p. 9) a interação leva ao compartilhamento, impulsiona os fluxos de informação e de conhecimento, que decorrem do movimento de uma rede e determinam seus vínculos. Marteleto e Silva (2004, p. 44) vão além, afirmando que as redes se constituem em canais pelos quais passam informação e conhecimento, devendo ser ampliadas para “criar ligações com outras comunidades semelhantes e assim ampliar o alcance de suas ações”.

Portanto, para compreender os fluxos de informação em redes sociais é fundamental investigar, ainda que de forma abrangente, tanto as ligações e interações dos atores de uma rede social específica quanto às inter-relações com comunidades semelhantes, pois os integrantes dessas redes mantêm contato com outras redes e espaços sociais. Dessa maneira, pressupõe-se que à medida que as interações entre os atores adjacentes e não adjacentes se modificam, também se alteram os fluxos de informação.

Quanto à forma de uma rede Castells (1999, p. 498) ressalta que a topologia definida por redes determina que a "distância (ou intensidade e frequência da interação) entre dois pontos (ou posições sociais) é menor (ou mais frequente, ou mais intensa), se ambos os pontos forem nós de uma rede do que se não pertencerem à mesma rede”.

Nessa mesma linha de pensamento, Albagli e Maciel (2004, p. 11) também advertem que as interações não devem ser estudadas apenas entre os agentes de uma mesma rede, mas sobretudo entre redes. Por outro lado, apontam que as interações locais permitem o desenvolvimento do conhecimento coletivo, e este pode ser diferenciado e desigualmente distribuído, podendo ou não constituir importante fonte de dinamismo para aquele ambiente. A localização ou proximidade espacial facilita a interação e a comunicação. No entanto, ela não é por si só um fator determinante para tal - são necessárias também condições institucionais e socioculturais que as favoreçam (ALBAGLI; MACIEL, 2004).

Em síntese, tem-se como desafio ampliar o escopo e a abrangência de análise das interações entre os atores locais da rede e destes com atores externos, a fim de elucidar o papel da proximidade territorial e da dinâmica socioinstitucional nessas interações (ALBAGLI; MACIEL, 2004). 
Uma outra, abordagem de rede é apontada por Tomaél (2008, p. 2) como “redes de conhecimento". Nessas redes, a informação carece de interpretação “e provém de um ator que coopera na rede com sua bagagem intelectual, cultural e organizacional.” Uma vez compartilhada por meio do conhecimento individual, esta informação pode contribuir para o desenvolvimento de parcerias que tragam benefícios recíprocos.

Tomaél (2008, p. 9) reflete ainda sobre a importância das redes de conhecimento para a interação e o compartilhamento da informação, mencionando que o termo redes de conhecimento é utilizado de forma ampla e inclui uma diversidade de modelos de trabalhos em cooperação. Para essa autora, as redes de conhecimento “estão vinculadas ao contexto que as gera, sendo o ambiente social e cultural, no qual elas proliferam, determinante para o seu direcionamento e evolução.”

Já Casarotto Filho e Pires (2001, p. 87) apresentam o conceito de redes de empresas como “conjunto de empresas interlaçadas por relacionamentos formais ou simplesmente negociais, podendo ou não ser circunscrito a uma região.”

Nessas redes, os nós podem ser as universidades, empresas industriais, pólos tecnológicos, instituições governamentais entre outros. Albagli e Maciel (2004) ressaltam que essas redes são ambientes que contribuem diretamente para que haja interação, troca de conhecimentos e aprendizado por meios diversos, tais como: a mobilidade local de trabalhadores, as redes formais e informais; e a existência de uma base social e cultural comum que dá o sentido de identidade e de pertencimento.

Ao estudar o fluxo de informação nas redes sociais é preciso considerar "as relações de poder que advêm de uma organização não-hierárquica e espontânea e procurar entender até que ponto a dinâmica do conhecimento e da informação interfere nesse processo” (MARTELETO, 2001, p. 73).

A contribuição teórica na tentativa de compreender os fluxos de informação nas redes sociais pode ser delineada a partir do estudo sobre o comportamento destes fluxos na rede social. Belkin (1978, p. 60) sugere que se considere um conjunto mínimo de requisitos para a definição de qualquer conceito científico e que podem ser classificados como: “metodológicos” que estão relacionados com a utilidade do conceito; “comportamentais”, 
que têm a ver com os fenômenos que o conceito deve explicar; e "definicionais" relacionados com o contexto do conceito.

Acredita-se que novas reflexões teóricas sobre fluxos de informação nas redes sociais possam ser explicitadas pela Ciência da Informação a partir dos requisitos “comportamentais” propostos por Belkin (1978), permitindo uma nova compreensão sobre este fenômeno.

\section{FLUXOS DE INFORMAÇÃO EM REDES SOCIAIS}

Ao estudar os fluxos de informação da rede, pode-se compreender como a informação flui, circula e é compartilhada, e como são constituídos os vínculos de interação para colaboração.

A informação e o conhecimento dão dinamismo às redes sociais, Tálamo (2004) afirma que a informação como fluxo, funciona para o sujeito como troca com o mundo exterior, o que lhe confere seu caráter social. A informação, quando “assimilada, interiorizada e processada por um sujeito específico é a base para sua integração no mundo, propiciando ajustes contínuos entre o mundo interior e o mundo exterior”.

Tálamo (2004) enfatiza que o modo pelo qual a sociedade integra-se aos fluxos de informação não se resume apenas à oferta da informação. “A informação circula socialmente e, para o sujeito, esta circulação deve ser percebida como conjunto de opções”.

No caso das redes sociais, acredita-se que os atores integram-se aos fluxos de informação quando reconhecem a existência de opções da informação que estão circulando na rede, selecionando as mais adequadas segundo o contexto em que se encontram. Deste modo, tão importante quanto à oferta da informação é a capacidade de percepção da informação como informação pelos atores, pois esta percepção pode tanto impulsionar os fluxos existentes quanto propiciar o desenvolvimento de novos fluxos na rede. 
Como assinalado por Barreto (1998) o fluxo de informação que, por meio de processos de comunicação realiza a intencionalidade do fenômeno da informação não objetiva somente uma passagem. Assim, ao atingir o público a que se destina, o fluxo pode

“[...] promover uma alteração; aqueles que recebem e podem elaborar a informação estão expostos a um processo de desenvolvimento, que permite acessar um estágio qualitativamente superior nas diversas e diferentes gradações da condição humana” (BARRETO, 1998, p. 123).

Esse desenvolvimento é repassado ao seu espaço de convivência. Tal espaço pode expressar-se em uma estrutura social em rede.

Uma vez que os fluxos de informação movimentam as redes, o direcionamento desses fluxos pode fortalecer e delinear uma rede, proporcionando sinergia às funções nela desdobradas. A informação mobiliza a rede e traz possibilidades de interação e expansão: "mediante o uso da informação, o estado existente modifica-se, expandindo o conhecimento que vai fortalecer o fluxo da informação e respaldar os processos individuais e coletivos” (TOMAÉL et al., 2005, p.102).

Uma das questões instigantes sobre redes sociais refere-se à compreensão da relação existente entre a estrutura da rede e o tipo de relacionamento decorrente desta estrutura. Pressupõe-se que a interação constante entre os atores e suas capacidades de intermediação impulsionam mudanças estruturais nos fluxos de informação, e elas se mantêm valendo-se das relações entre diversos indivíduos dentro do ambiente em redes.

Em relação às interações em que a troca é a informação, Tomaél; Alcará, Di Chiara (2005, p. 96) defendem que

a mudança estrutural que pode ser percebida é a do conhecimento, quanto mais informação trocamos com o ambiente que nos cerca, com os atores da nossa rede, maior será nossa bagagem de conhecimento, maior será nosso estoque de informação.

É nesse conjunto de significados que as redes sociais estão inseridas.

Nesse sentido, Wellman (1996) afirma que a identidade peculiar da rede em determinada situação, ou seja, a representação e a interpretação das relações estão particularmente relacionadas à realidade que a cerca, sendo a rede influenciada pelo seu contexto e esse por ela.

(c) Revista Digital de Biblioteconomia e Ciência da Informação,Campinas, v.7, n. 2, p. 102-117, jan./jun. 2010- ISSN: 1678-765X. 
Tal fato tem importantes implicações já que o movimento das redes é ditado por interações representadas por relações sociais, culturais, econômicas, políticas, entre outras. Além disso, como afirma Marteleto (2001, p. 75) “toda rede tem um alto grau de mutabilidade”.

Para Tomaél; Alcará, Di Chiara (2005, p.102) dependendo dos interesses dos indivíduos, a rede pode ser seccionada em grupos que geralmente são profícuos para a própria rede, mobilizando atores que estejam envolvidos com uma temática específica. A rede “[...] favorece as ligações entre atores com o poder de direcionar os fluxos de informação a indivíduos que partilham de interesses comuns, proporcionando maiores condições para a inovação” (TOMAÉL; ALCARÁ, DI CHIARA, 2005, p.102).

Dentre os trabalhos recentes sobre fluxos e transferência de informação na área da Ciência da Informação, destaca-se o realizado por Marteleto (2001) intitulado “Análise de redes sociais - aplicação nos estudos de transferência da informação” que teve como foco as redes de movimentos sociais na região da Leopoldina, subúrbio do Rio de Janeiro. Nesse trabalho, a rede foi seccionada em grupos de atores envolvidos com a temática fundamentada nos princípios e práticas de educação popular e saúde, objetivando compreender a informação e a comunicação como instrumentos de mobilização nos movimentos sociais locais, utilizando a metodologia de análise de redes sociais.

Como destaca Marteleto (2001, p. 79) o enfoque das redes aliado ao uso da metodologia de análise de redes sociais e da análise qualitativa, permitiu elucidar que esses movimentos estão colocando em prática uma nova compreensão do conhecimento e da informação. Vale destacar que Marteleto (2001) utilizou o software de Análise de Redes UCINET 5.0, por meio do qual foram exploradas as medidas denominadas "cliques" e “centralidade” - visando compreender os papéis que cada ator desempenhava. Porém, existem outras medidas de ARS que podem ser utilizadas e atribuídas aos atores, com o intuito de compreender os fluxos de informação, conforme será proposto a seguir. 


\section{Análise de Redes Sociais: Apresentação do Método}

A metodologia de Análise de Redes Sociais será utilizada no que concerne aos elementos a serem utilizados para identificar os atores da rede que mantêm relações mais estreitas entre si e assim permitindo que a informação circule pelo ambiente da rede. Também possibilitará mapear e descrever os atores que atuam na mediação das trocas facilitando o fluxo de informação na rede, bem como propor uma nova compreensão dos fluxos de informação que otimize as relações dentro do ambiente em que as redes se movimentam.

A análise de redes sociais (ARS) é uma metodologia oriunda da sociologia, da psicologia social e da antropologia, e tem sido utilizada com o objetivo de estudar as ligações entre atores sociais. Nesse tipo de análise, o termo atores é utilizado para se referir tanto às pessoas - consideradas como unidades individuais - como às unidades sociais coletivas como, por exemplo, departamentos dentro de uma organização, agências de serviço público em uma cidade, países de um continente ou mundo (WASSERMAN; FAUST, 1999).

O método de análise de redes sociais pode ser considerado um método quantitativo, já que utiliza a base matemática e estatística para visualizar as redes e sistematizar a informação de forma a possibilitar a visualização de sua estrutura e seus padrões (SOUSA, 2007).

Tomaél, Alcará e Di Chiara (2005, p. 85) destacam o aspecto qualitativo desse método já que este permite "investigar as aspirações, atitudes, crenças, valores e reflexos que os padrões de relacionamentos produzem no contexto em que se desenvolvem”.

Richardson (1999) enfatiza que o método quantitativo caracteriza-se pelo emprego da quantificação tanto nas modalidades de coleta de informações, quanto no tratamento delas por meio de técnicas estatísticas. Já a abordagem qualitativa, justifica-se, sobretudo, por ser uma forma para entender a natureza de um fenômeno social.

Como ressalta Marteleto (2001, p. 72) a análise de redes é “[...] o meio para realizar uma análise estrutural cujo objetivo é mostrar em que a forma da rede é explicativa dos fenômenos analisados”. O uso dessa metodologia em estudos da área da Ciência da 
Informação pode, por exemplo, ajudar a compreender os fenômenos dos fluxos de informação a partir das redes sociais.

Para Degenne e Forse (1994, p. 7) citado por Marteleto (2001, p. 72), esta metodologia busca demonstrar que a análise de uma díade (interação entre duas pessoas) só tem sentido em relação ao conjunto das outras díades da rede, porque a sua posição estrutural influência sua forma, seu conteúdo e sua função. Neste contexto, pode-se inferir que a posição estrutural dos elos (atores) afeta os fluxos de informação na rede e o trajeto que a informação pode percorrer.

Com o intuito de descrever a estrutura e as relações ou ligações de uma rede, Wasserman e Faust (1994) sugerem que sejam definidas as “variáveis estruturais” que são aquelas relacionadas às características dos atores da rede, e as “variáveis de composição” que mostram o tipo de ligação existente entre pares de atores.

Além disso, é importante definir o grupo no qual essas variáveis serão medidas, para posterior identificação do vínculo relacional entre os atores - "relation tie" - no que se refere ao direcionamento, ou seja, ao sentido da relação ou caminho, que neste caso pode ser - direcional - quando a ligação entre dois atores possui uma origem e um destino, ou não-direcional - quando a ligação não mostra sentido.

Wasserman e Faust (1994) ressaltam que os dados das redes sociais podem ser estudados tendo como base as unidades de observação, com uso de entrevistas, observações e questionário para mapear a interação dos atores entre si; a modelagem; e a quantificação das relações. As diferentes abordagens estão sumarizadas no Quadro 1: 
Quadro 1: Abordagens sobre os estudos dos dados das redes sociais

\begin{tabular}{ll}
\hline ABORDAGEM & RELAÇÃO DE INTERAÇÃO \\
\hline Unidade de observação & $\begin{array}{l}\text { atores, pares de atores e ligações } \\
\text { relacionais }\end{array}$ \\
Unidade de modelagem & ator, díade, tríade, subgrupo e a rede \\
Quantificação das relações & direcional e não-direcional \\
\hline
\end{tabular}

Fonte: WASSERMAN; FAUST, 1994.

\subsection{Coleta de dados em redes sociais}

Nas redes sociais, as pesquisas geralmente são conduzidas a partir de uma população identificada e não de uma amostra. Hannemann e Riddle (2005) citado por Sousa (2007, p.133) explicam que isto ocorre porque ao selecionar um ator, o mesmo poderá escolher outros atores de seu relacionamento que podem não estar na amostra, já que um ator pode atuar como intermediário ligando outros que não estão diretamente conectados. Neste contexto, Hannemann e Riddle (2005) propõem os seguintes métodos de escolha:

- método da rede total: coleta-se informações das relações de todos os atores da rede, utilizado na medição e descrição dos conceitos estruturais da rede;

- método da bola de neve: a coleta inicia-se a partir de um ator ou grupo, e se estende a outros a partir destes. Cada ator da lista inicial identifica um conjunto de outros atores que possui ligações, formando uma segunda lista. Estes por sua vez, identificam outros atores que possuem ligações, e assim sucessivamente até que não sejam encontrados novos atores ou até uma parada proposital. Este método é utilizado quando se deseja identificar redes de contatos de negócios e outros tipos de estrutura;

- rede ego com conexões "amigas”: seleciona-se um ator ou atores e em seguida é realizada a identificação de atores que pertencem à sua rede. Em seguida, identificam-se quais os atores estão conectados entre si. É adotado quando se almeja trabalhar com redes pequenas pelo fato de disponibilizar poucas informações sobre a rede como um todo;

- rede ego: quando o objetivo é a coleta de informações de um ator e seus pares sem estabelecer as ligações entre os pares.

Quanto aos instrumentos que podem ser utilizados para a coleta de dados na análise de redes, Wasserman e Faust (1994) destacam: os questionários; as entrevistas; a observação; os arquivos e outras técnicas. A informação obtida por meio dos 
questionários permite observar as características de um indivíduo ou grupo beneficiando a análise a ser feita pelo pesquisador (RICHARDSON, 1999, p.189).

Wasserman e Faust (1994) relacionam diferentes tipos de questionários que podem ser utilizados na coleta de dados em Análise de Redes Sociais. E os categorizam em: questionário lista; identificação livre; escolha livre; escolha fixa; e avaliação.

Quadro 2: Tipos de questionários para coleta de dados na Análise de Redes Sociais.

\begin{tabular}{|c|c|}
\hline $\begin{array}{c}\text { TIPOS DE } \\
\text { QUESTIONÁRIOS }\end{array}$ & APLICAÇÃO \\
\hline Lista & $\begin{array}{l}\text { o pesquisador conhece todos os atores do grupo e oferece a } \\
\text { lista para o ator-alvo. }\end{array}$ \\
\hline Identificação Livre & $\begin{array}{l}\text { o respondente nomeia os atores com os quais possui } \\
\text { ligações. }\end{array}$ \\
\hline Escolha Livre & o repondente indica todos os nomes que ele encontrar \\
\hline Escolha Fixa & $\begin{array}{l}\text { o questionário estabelece a quantidade máxima de atores } \\
\text { que o respondente deve nomear }\end{array}$ \\
\hline $\begin{array}{l}\text { Avaliação ou Classificação } \\
\text { Completa }\end{array}$ & $\begin{array}{l}\text { produz escalas de valores para as ligações entre os atores } \\
\text { da rede. A avaliação atribui um valor que representa uma } \\
\text { categorização, uma classificação }\end{array}$ \\
\hline
\end{tabular}

Fonte: Adaptado de WASSERMAN; FAUST (1994) citado por SOUZA (2007).

\subsection{Medidas e Métricas em Redes Sociais}

Na análise de redes sociais, as medidas e métricas relacionadas aos atores podem ser utilizadas visando compreender os fluxos de informação das redes. Tais métricas são disponibilizadas no software do UCINET, que permite estabelecer um modelo para a análise dos dados quantitativos e qualitativos anteriormente coletados e escolher as métricas que se aderem ao objeto em estudo. 
Acredita-se que as métricas de proximidade, intermediação, centralidade e gatekeeper ajudam a identificar e compreender quais atores atuam como fonte de informação para os demais e como a estrutura da rede afeta o fluxo de informação. O Quadro 3 traz algumas definições das medidas da ARS atribuídas aos atores.

Quadro 3: Medidas de Análise de Redes Sociais atribuídas aos atores.

\begin{tabular}{l|l|}
\hline Grau (Degree) & Número de ligações diretas com outros atores. \\
\hline Grau de entrada (In-degree) & Número de ligações direcionais que um ator recebe. \\
\hline Grau de saída (Out-degree) & Número de ligações direcionais de um ator para outros. \\
\hline Proximidade (Closeness) & $\begin{array}{l}\text { Medida que indica o quão um ator está próximo ou pode alcançar } \\
\text { outros atores na rede. }\end{array}$ \\
\hline Intermediação (Betweenness) & $\begin{array}{l}\text { Medida do quão um ator exerce papel de mediador ou está entre dois } \\
\text { atores em um caminho mais curto. }\end{array}$ \\
\hline Centralidade (Centrality) & Medida do quão central um determinado ator está na rede. \\
\hline Prestígio (Prestige) & Semelhante à centralidade, porém é baseado em relações direcionais. \\
\hline Ponte (Bridge) & Ator que é membro de dois ou mais grupos. \\
\hline Gatekeeper & Ator de medida ou controla o fluxo entre uma parte da rede e outra. \\
\hline Isolado (isolate) & Ator que não possui relações ou poucas relações com outros atores. \\
\hline Sousa (2007) & \\
\hline
\end{tabular}

Embora a análise quantitativa dos resultados corrobore a compreensão dos fluxos de informação, Cross e Parker (2004) sugerem a criação de sessões de discussão sobre os resultados - reuniões - visando melhor entendimento quando eles não revelarem de maneira clara uma determinada dinâmica que ocorre na rede. Essas reuniões podem ser complementadas com entrevistas para a confirmação dos resultados, sendo realizadas com as pessoas centrais da rede ou ainda com pessoas de determinados departamentos.

Acredita-se, que, as relações e ligações entre os atores das redes definem e direcionam os fluxos de informação. Sob este olhar, o posicionamento da Ciência da Informação como facilitadora de estudos sobre essa temática pode representar um anseio da comunidade de atores de redes sociais que elaboram as suas práticas cotidianas e estratégias de atuação a partir dos fluxos de informação. 


\section{CONSIDERAÇÕES FINAIS}

O estudo sobre o "caminho" percorrido pela informação na rede social e o modo como ocorrem os fluxos de informação pode resultar em novas reflexões conceituais em relação à estrutura deste fluxo e aos vínculos relacionais que se configuram e re-configuram na rede.

A necessidade de compreender os fluxos de informação, seus fatores, possíveis desdobramentos e perspectivas têm motivado a atenção às interações em redes considerando-as, sobretudo, como ambientes sociais e não apenas espaços econômicos.

A Ciência da Informação pode propor conceitos em cuja pluralidade poderia identificar maneiras de ver e interpretar o fenômeno dos fluxos de informação nas redes sociais.

Sugere-se, portanto, que sejam analisados os fluxos de informação segundo o contexto de cada ator, para a proposição de reflexões conceituais científicas aderentes e alinhadas à Ciência da Informação.

\section{REFERÊNCIAS}

ALBAGLI, S.; MACIEL, M. L. Informação e conhecimento na inovação e no desenvolvimento local. Ciência da Informação, Brasília, v. 33, n.3, p. 9-16, set./dez. 2004.

. Globalização e espacialidade: o novo papel do local. In: CASSIOLATO, J. E.; LASTRES, H. M. M. Globalização e inovação localizada: experiências de sistemas locais no Mercosul. Brasília: IBICT/IEL, 1999.

BARRETO, A. Mudança estrutural no fluxo do conhecimento: a comunicação eletrônica. Ciência da Informação, Brasília, v. 27, n. 2, 1998.

BELKIN, N. Information concepts for information science. Journal of Documentation, v. 34, n.1, mar. 1978.

CASSAROTTO, F. N.; PIRES, L. H. Redes de pequenas e médias empresas e desenvolvimento local: estratégias para a conquista da competitividade global com base na experiência italiana. São Paulo: Atlas, 2001.

CASTELLS, M. A sociedade em rede: a era da informação: economia, sociedade e cultura. São Paulo: Paz e Terra, 1999. v.1.

CROSS, R. L; PARKER, A. The hidden power of social networks: understanding how work really gets done in organizations. Harvard Business School Press, 2004.

(c) Revista Digital de Biblioteconomia e Ciência da Informação,Campinas, v.7, n. 2, p. 102-117, jan./jun. 2010- ISSN: 1678-765X. 
DEGENNE, A.; FORSÉ, M. Les réseaux sociaux: une analyse structurale en sociologic. Paris: Armand Colin, 1994.

HANNEMAN, R. A.; RIDDLE, M. Introduction to social network methods. Riiverside: University of California. Disponível em: http://faculty.ucr.edu/ hanneman/). Acesso em: 15 set. 2005.

MARTELETO, R. M. Análise de redes sociais: aplicação nos estudos de transferência da informação. Ciência da Informação, Brasília, v.30, n.1, p.71-81, jan./abr. 2001.

.; SILVA, A. B. O. Redes e capital social: o enfoque da informação para o desenvolvimento local. Ciência da Informação, Brasília, v.33, n.3, p.41-49, set./dez. 2004.

POLO TECNOLÓGICO TÊXTIL DE AMERICANA/POLOTECTEX. Disponível em: http://www.polotectex.com.br/index.php?option=com_content\&task=view\&id=58\&Itemi $\underline{\mathrm{d}=83}$. Acesso em: 10 ago. 2008

RICHARDSON, R. J. Pesquisa social: métodos e técnicas. São Paulo: Atlas, 1999.

SHERA, J. H.; CLEVELAND, D. B. History and foundations of information science. Annual Review of Information Science and Technology, v.12, p. 249-275, 1977.

SOUSA, P. T. C. Metodologia de análise de redes sociais. In: MULLER, S. P. M. (Org.). Métodos para a pesquisa em Ciência da Informação. Brasília: Thesaurus, 2007.

TALAMO, M. F. G. M. A pesquisa: recepção da informação e produção do conhecimento. DataGramaZero: revista de ciência da informação, Rio de Janeiro, v. 5, n. 2, abr. 2004. Disponível em: <http:www.dgzero.org> Acesso em: 24 maio 2008.

TOMAÉL, M. I. Redes de conhecimento. DataGramaZero: revista de ciência da informação, Rio de Janeiro, v. 9, n. 2, abr. 2008.

.; ALCARÁ, A. R.; DI CHIARA, I. G. Das redes sociais à inovação. Ciência da Informação, Brasília, v.34, n.2, maio/ago. 2005.

WASSERMAN, S.; FAUST, K. Social network analysis: methods and applications. In: . Structural analysis in social the social science series. Cambridge: Cambridge University Press, 1994. 857p.

WELLMAN, B. Are personal communities local?: a dumpatarian reconsideration. Social Networks, Amsterdam, v.18, p.347-354, 1996. 


\section{Cibele Roberta Sugahara}

Doutoranda do Programa de Pós-Graduação em Ciência da Informação da Escola de Comunicação e Artes da Universidade de São Paulo. cibelesu@yahoo.com.br

\section{Waldomiro Vergueiro}

Professor Titular, Chefe do Departamento de Biblioteconomia e Documentação da Escola de Comunicações e Artes da Universidade de São Paulo. wdcsverg@usp.br

Recebido em: 24/06/2009

Aceito para publicação em: jul/2009 